Volume 26 (2019) 41-52

DOI: $10.24330 /$ ieja. 586920

\title{
CLASS LENGTH OF ELEMENTS OF GROUP IN THE NORMALIZED UNIT GROUP
}

\author{
S. Kaur and M. Khan \\ Received: 1 June 2018; Accepted: 19 May 2019 \\ Communicated by Abdullah Harmancı
}

\begin{abstract}
Let $F$ be a finite field of characteristic $p>0$. In this article, we obtain a relation between the class length of elements of a finite $p$-group $G$ in the normalized unit group $V(F G)$ and its unitary subgroup $V_{*}(F G)$, when $p$ is an odd prime. We also provide the size of the conjugacy class of non-central elements of a group $G$ in $V(F G)$, where either $G$ is any finite $p$-group with nilpotency class 2 or $G$ is a $p$-group with nilpotency class 3 such that $|G| \leq p^{5}$.
\end{abstract}

Mathematics Subject Classification (2010): 16U60, 20C05, 20E45

Keywords: Group ring, unit group, unitary units, conjugacy class

\section{Introduction}

Let $F G$ be the group algebra of a finite $p$-group $G$ over a field $F$ of characteristic $p$. The augmentation ideal of $F G$, denoted by $\omega(F G)$, is set of all the elements of $F G$ with augmentation 0 . The elements of the set

$$
V(F G)=\left\{\sum_{g \in G} a_{g} g \in \mathcal{U}(F G) \mid \sum_{g \in G} a_{g}=1\right\}
$$

form a subgroup known as the normalized unit group of $F G$. The extension of the trivial homomorphism $G \rightarrow\{e\}$ to algebra homomorphism from $F G$ onto $F$ has kernel $\omega(F G)$. Since $\omega(F G)$ is a nilpotent ideal, the algebra homomorphism induces an epimorphism from $\mathcal{U}(F G)$ onto $\mathcal{U}(F)$ with kernel $(1+\omega(F G))$ which is same as $V(F G)$. Therefore, $V(F G)$ is a $p$-group of order $|F|^{(|G|-1)}$. The understanding of the structure of this class of $p$-groups is very interesting problem in the theory of modular group algebras.

The conjugacy classes in a non-abelian group provide information about its structure. Since the character induced by a representation of a group is a class function, the knowledge of the conjugacy classes of the group facilitates the computation of its character table.

The study of the conjugacy classes of the normalized unit groups of modular group algebras was initiated by D. B. Coleman in [6]. In this paper, he proved that 
if some conjugacy class of $V(F G)$ contains an element of $G$, then its intersection with $G$ is a conjugacy class in $G$. Furthermore, several results regarding the length of conjugacy classes of $V(F G)$ have been obtained in the last decade.

Rao and Sandling proved in [7] that $p$ can never occur as the cardinality of any conjugacy class of $V\left(F_{p} G\right)$. Then A. A. Bovdi and C. Polcino Milies established $|F|^{2}$ as the lower bound for the size of any non-singleton conjugacy class in $V(F G)$ (see [3]). If $G$ is a finite $p$-group which has a quotient with commutator subgroup of order $p$ and centre of index $p^{k}$, then A. Bovdi, L. G. Kovacs and S. Mihovski provided an element of $V(F G)$ whose class length is $|F|^{k}$ (see [5]). The problem of finding the length of the conjugacy classes in unit groups of group algebras has also been studied in [1] and [2].

Let $v$ be an element of $V(F G)$. Throughout the paper, $C_{F G}(v)$ denotes the centralizer of $v$ in $F G$ and $C_{v}$ denotes the conjugacy class of $v$ in $V(F G)$. If $H$ is a subgroup of $G$ and $\left\{e=c_{1}, c_{2}, \ldots, c_{l}\right\}$ is a left transversal of $H$ in $G$, then $F G=F H \oplus M$ as an $F$-module, where $M=\bigoplus_{i=2}^{l} c_{i} F H$.

The anti-automorphism $g \mapsto g^{-1}$ of $G$ can be extended linearly to an involution * of $F G$. The restriction of * to $V(F G)$ followed by the map $v \mapsto v^{-1}$ gives an automorphism of $V(F G)$. The normalized units which remain fixed under this automorphism are called unitary units and they form a subgroup of $V(F G)$ which is called the unitary subgroup $V_{*}(F G)$.

In the recent paper, we provide the length of the conjugacy class of elements of a group $G$ in the normalized unit group $V(F G)$ and in $V_{*}(F G)$, where $G$ is a finite $p$-group and $F$ is a finite field of characteristic $p$. Let $v$ be an element of $V(F G)$. Then $C_{F G}(v)=F \oplus C_{\omega(F G)}(v)$, where $C_{\omega(F G)}(v)$ is the centralizer of $v$ in $\omega(F G)$. Therefore, the length of the conjugacy class $C_{v}$ of $v$ in $V(F G)$ is given by

$$
\left|C_{v}\right|=\frac{|V(F G)|}{\left|C_{V(F G)}(v)\right|}=\frac{|F|^{|G|-1}}{\left|C_{\omega(F G)}(v)\right|}=|F|^{|G|-\left(\operatorname{dim}_{F}\left(C_{F G}(v)\right)\right)} .
$$

In Section 2, we study the class length of elements of a finite $p$-group $G$ in $V_{*}(F G)$, where $p$ is an odd prime. The main result of this section is as follows:

Theorem 1.1. Let $F$ be a finite field of characteristic $p$ and $G$ be a finite p-group, where $p$ is an odd prime. For a non-central element $g \in G$, let $C_{g}^{*}$ denote the conjugacy class of $g$ in $V_{*}(F G)$. Then

$$
\left|C_{g}\right|=\left|C_{g}^{*}\right|^{2}
$$


As a corollary, we obtain the length of the conjugacy class of elements of $G$ in $V(F G)$, where $G$ is a finite $p$-group of nilpotency class 3 and the commutator subgroup $G^{\prime}$ of $G$ is elementary abelian.

In Section 3, the size of the conjugacy class of any non-central element of $G$ in $V(F G)$ is obtained, where $G$ is a finite $p$-group with nilpotency class 2 . The main theorem of this section is as follows:

Theorem 1.2. Let $F$ be a finite field of characteristic $p$ and $G$ be a finite p-group with nilpotency class 2 . Let $g$ be a non-central element of $G$. If $\left\{e=c_{1}, c_{2}, \ldots, c_{l}\right\}$ is a transversal of $G^{\prime}$ in $G$ and $n_{i}=\left[G^{\prime}:\left\langle\left(c_{i}, g\right)\right\rangle\right]$, then

$$
\left|C_{g}\right|=|F|^{|G|-\left(\left|G^{\prime}\right|+\sum_{i=2}^{l} n_{i}\right)} .
$$

In particular, we have:

Theorem 1.3. Let $F$ be a finite field of characteristic $p$ and $G$ be a non-abelian group of order $p^{3}$. Let $g$ be any non-central element of $G$. Then

$$
\left|C_{g}\right|=|F|^{p(p-1)^{2}} .
$$

In Section 4, we provide the cardinality of the conjugacy class of elements of $G$ in $V(F G)$, where $G$ is a group of order $p^{4}$ or $p^{5}$ such that $p$ is an odd prime and nilpotency class of $G$ is 3 . The following theorems constitute the main results of this section:

Theorem 1.4. Let $p$ be an odd prime and $F$ be a finite field of characteristic $p$. Let $G$ be a group of order $p^{4}$ with nilpotency class 3 . If $g$ is a non-central element of $G$, then

$$
\left|C_{g}\right|=\left\{\begin{array}{lr}
|F|^{(p-1)^{2} p^{2}}, & \text { if }\left|C_{G}(g)\right|=p^{3} \\
|F|^{(p-1)^{2} p(p+1)}, & \text { otherwise. }
\end{array}\right.
$$

Theorem 1.5. Let $p$ be an odd prime and $F$ be a finite field of characteristic $p$. Let $G$ be a group of order $p^{5}$ with nilpotency class 3 such that $\frac{G}{G^{\prime}}$ is an elementary abelian group. If $g$ is a non-central element of $G$, then

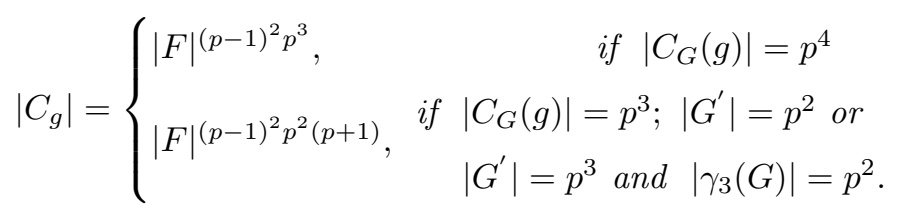

Theorem 1.6. Let $p$ be an odd prime and $F$ be a finite field of characteristic $p$. Let $G$ be a group of order $p^{5}$ such that

$$
\frac{G}{G^{\prime}} \cong C_{p^{2}} \times C_{p}
$$


If $g$ is a non-central element of $G$, then

$$
\left|C_{g}\right|= \begin{cases}|F|^{(p-1)^{2} p^{3},} & \text { if }\left|C_{G}(g)\right|=p^{4} \\ |F|^{(p-1)^{2} p^{2}(p+1)}, & \text { if }\left|C_{G}(g)\right|=p^{3} \text { and } G^{\prime} \cong C_{p} \times C_{p} \\ |F|^{p^{2}\left(p^{3}-3 p+2\right)}, & \text { if }\left|C_{G}(g)\right|=p^{3} \text { and } G^{\prime} \cong C_{p^{2}} .\end{cases}
$$

\section{On the length of conjugacy class of elements of group $G$ in $V(F G)$ and $V_{*}(F G)$}

Let $p$ be an odd prime. Let $F$ be a finite field of characteristic $p$ and $G$ be a finite $p$-group. In this section, we obtain that the length of the conjugacy class of an element of $G$ in the normalized unit group $V(F G)$ is the square of the size of its conjugacy class in the unitary subgroup $V_{*}(F G)$.

Let

$$
S_{1}=\left\{x \in \omega(F G) \mid x^{*}=x\right\}
$$

and

$$
S_{2}=\left\{x \in \omega(F G) \mid x^{*}=-x\right\}
$$

be the set of symmetric and skew-symmetric elements of $\omega(F G)$, respectively. Clearly, $\omega(F G)=S_{1} \oplus S_{2}$. It is known (see [4]) that corresponding to each skewsymmetric element $k \in S_{2}$, the element $u=(1-k)(1+k)^{-1} \in V_{*}(F G)$. Moreover, for any $u \in V_{*}(F G)$, the element $k=(1-u)(1+u)^{-1}$ is skew-symmetric. Further, if $v=\sum_{g \in G} \alpha_{g} g$ is a skew-symmetric element of $\omega(F G)$, then $v=\sum_{g \in G} \alpha_{g}\left(g-g^{-1}\right)$. Hence $\left|S_{2}\right|=\left|V_{*}(F G)\right|=|F|^{\frac{|G|-1}{2}}$.

In order to obtain the main result, we shall make use of the following lemmas:

Lemma 2.1. Let $F$ be a finite field of characteristic $p$ and $G$ be a finite p-group, where $p$ is an odd prime. Then for any element $v \in V(F G)$,

$$
\left|C_{V_{*}(F G)}(v)\right|=\left|C_{S_{2}}(v)\right| .
$$

Proof. Assume that $C_{S_{2}}(v)=\left\{y_{1}, y_{2}, \ldots, y_{n}\right\}$. Note that for each $y_{i} \in C_{S_{2}}(v)$, the element $u_{i}=\left(1-y_{i}\right)\left(1+y_{i}\right)^{-1} \in C_{V_{*}(F G)}(v)$. Therefore, $\left|C_{V_{*}(F G)}(v)\right| \geq n$. Further, assume that $u \in C_{V_{*}(F G)}(v)$ such that $u \neq u_{i}$, for any $1 \leq i \leq n$. Then $k=(1-u)(1+u)^{-1} \in C_{S_{2}}(v)$. Hence $k=y_{i}$, for some $i$ and so $(1-k)(1+k)^{-1}=$ $u=u_{i}$, which is a contradiction. Thus $\left|C_{V_{*}(F G)}(v)\right|=n$.

Lemma 2.2. Let $F$ be a finite field of characteristic $p$ and $G$ be a finite p-group, where $p$ is an odd prime. Then for any element $v \in V_{*}(F G)$,

$$
\left|C_{V(F G)}(v)\right|=\left|C_{S_{1}}(v)\right|\left|C_{S_{2}}(v)\right| .
$$


Proof. Define a map $\phi: C_{\omega(F G)}(v) \rightarrow C_{S_{1}}(v)$ by the rule $w \mapsto \frac{w+w^{*}}{2}$. Clearly, it is an $F$-module epimorphism with $\operatorname{ker}(\phi)=C_{S_{2}}(v)$.

Proof of Theorem 1.1: Let $\alpha=\sum_{h \in G} \alpha_{h}\left(h-h^{-1}\right) \in C_{S_{2}}(g)$. Then write

$$
\alpha=\sum_{h \in C_{G}(g)} \alpha_{h}\left(h-h^{-1}\right)+\beta, \text { where } \beta=\sum_{h \in G \backslash C_{G}(g)} \alpha_{h}\left(h-h^{-1}\right) .
$$

Consider the automorphism $\phi_{g}: G \rightarrow G$ defined by $h \mapsto g^{-1} h g$. Let $H$ be the cyclic group generated by $\phi_{g}$. Consider the action of $H$ on $G$ induced by the automorphism $\phi_{g}$. Since $g^{-1} \alpha g=\alpha$, we get that $\operatorname{supp}(\beta)$ is closed under this action and the coefficients agree on the orbits formed under this action. Let $O_{h}$ denote the orbit of any $h \in G \backslash C_{G}(g)$. Assume that $\operatorname{supp}(\beta)=\bigcup_{i=1}^{n} O_{h_{i}}$. Since $G$ is a group of odd order, $h_{i}$ and $h_{i}^{-1}$ are in different orbits and so

$$
\beta=\sum_{i=1}^{\frac{n}{2}} \alpha_{h_{i}}\left(\widehat{O_{h_{i}}}-\widehat{O_{h_{i}^{-1}}}\right) .
$$

Note that the elements $\beta_{i}=\alpha_{h_{i}}\left(\widehat{O_{h_{i}}}\right)$ and $\beta_{i}^{\prime}=\alpha_{h_{i}}\left(\widehat{O_{h_{i}^{-1}}}\right)$ commute with $g$. Hence the element

$$
\alpha^{\prime}=\sum_{h \in C_{G}(g)} \alpha_{h}\left(h+h^{-1}-2\right)+\sum_{i=1}^{\frac{n}{2}} \alpha_{h_{i}}\left(\widehat{O_{h_{i}}}+\widehat{O_{h_{i}^{-1}}}-2\right)
$$

is in $C_{S_{1}}(g)$. Similarly, corresponding to each element of $C_{S_{1}}(g)$, we can obtain an element in $C_{S_{2}}(g)$. Therefore, $\left|C_{S_{1}}(g)\right|=\left|C_{S_{2}}(g)\right|$. Hence Lemma 2.1 and Lemma 2.2 imply that $\left|C_{V(F G)}(g)\right|=\left|C_{V_{*}(F G)}(g)\right|^{2}$. Thus

$$
\left|C_{g}\right|=\frac{|V(F G)|}{\left|C_{V(F G)}(g)\right|}=\frac{\left|V_{*}(F G)\right|^{2}}{\left|C_{V_{*}(F G)}(g)\right|^{2}}=\left|C_{g}^{*}\right|^{2} .
$$

Corollary 2.3. Let $F$ be a finite field of characteristic $p$ and $G$ be a finite p-group, where $p$ is an odd prime. Let $g$ be a non-central element of $G$ such that $g^{p} \in Z(G)$. Then

$$
\left|C_{g}\right|=|F|^{\frac{(p-1)}{p}\left(|G|-\left|C_{G}(g)\right|\right)} .
$$

Proof. Since $g^{p} \in Z(G)$, corresponding to any $h \in G \backslash C_{G}(g)$ the orbit $O_{h}=$ $\left\{g^{-i} h g^{i} \mid 0 \leq i \leq p-1\right\}$. Therefore, each non-singleton orbit has size $p$ and hence the elements of the set $G \backslash C_{G}(g)$ can be written as the disjoint union of the orbits 
$O_{h_{i}}$ and $O_{h_{i}^{-1}}$ for $1 \leq i \leq m$, where $m=\frac{|G|-\left|C_{G}(g)\right|}{p}$. Then any element of $C_{S_{2}}(g)$ is of the from

$$
\sum_{h \in C_{G}(g)} \alpha_{h}\left(h-h^{-1}\right)+\sum_{i=1}^{m} \alpha_{h_{i}}\left(\widehat{O_{h_{i}}}-\widehat{O_{h_{i}^{-1}}}\right) .
$$

Hence $\left|C_{S_{2}}(g)\right|=\left|C_{V_{*}}(g)\right|=|F| \frac{\left|C_{G}(g)\right|-1}{2}+\frac{|G|-\left|C_{G}(g)\right|}{{ }^{2} p}$ and therefore, we obtain the desired result.

If the nilpotency class of $G$ is 3 , then we have the following result:

Lemma 2.4. Let $G$ be a finite p-group of class 3. For any two elements $g, h \in G$,

$$
\left(g^{n}, h\right)=(g, h)^{n}((g, h), g)^{\left(\begin{array}{c}
n \\
2
\end{array}\right)}, \text { for } n \geq 2 .
$$

Proof. The result can be obtained by induction.

Moreover, if the commutator subgroup $G^{\prime}$ of $G$ is elementary abelian, then we can obtain the length of the conjugacy class of any element of $G$ in $V(F G)$.

Corollary 2.5. Let $F$ be a finite field of characteristic $p$ and $G$ be a finite $p$-group of nilpotency class 3 , where $p$ is an odd prime. If the commutator subgroup $G^{\prime}$ of $G$ is elementary abelian, then for any non-central element $g \in G$,

$$
\left|C_{g}\right|=|F|^{\frac{(p-1)}{p}\left(|G|-\left|C_{G}(g)\right|\right)} .
$$

Proof. Let $h$ be any element of $G$. By Lemma $2.4,\left(g^{p}, h\right)=(g, h)^{p} z^{\left(\begin{array}{c}p \\ 2\end{array}\right)}$, where $z \in \gamma_{3}(G)$. Since $G^{\prime}$ is elementary abelian, we get that $\left(g^{p}, h\right)=e$ and hence $g^{p} \in Z(G)$. Then using the same argument as in the previous corollary, we get the result.

\section{Class size of the elements of finite $p$-group $G$ of class 2 in $V(F G)$}

Let $F$ be a finite field of characteristic $p$ and $G$ be a finite $p$-group with the lower central series

$$
G=\gamma_{1}(G) \supseteq \gamma_{2}(G) \supseteq \ldots \supseteq \gamma_{c-1}(G) \supseteq \gamma_{c}(G) \supseteq \gamma_{c+1}(G)=\{e\} .
$$

In this section, firstly we provide the size of the centralizer of any non-central element of $G$ in the subring $F \gamma_{c-1}(G)$ of $F G$. Then we aim at obtaining the size of the conjugacy classes of elements of any nilpotent group $G$ of class 2 in $V(F G)$. In this direction, the following lemmas play a very crucial role. 
Lemma 3.1. Let $F$ be a field and $G$ be a group. Let $H$ be a normal subgroup of $G$. If $g$ is a non-central element of $G$, then

$$
C_{F G}(g)=C_{F H}(g) \oplus C_{M}(g),
$$

where $F G=F H \oplus M$ as an $F$-module.

Proof. Clearly, $C_{F H}(g) \oplus C_{M}(g) \subseteq C_{F G}(g)$. Let $y \in C_{F G}(g)$ such that $y=\beta+\gamma$, where $\beta \in F H$ and $\gamma \in M$. Consider the projection map

$$
\pi_{H}: F G \rightarrow F H
$$

defined as

$$
\sum_{g \in G} a_{g} g \mapsto \sum_{g \in H} a_{g} g
$$

Therefore, $\pi_{H}(y)=\beta$. Further, note that $\operatorname{supp}\left(g^{-1} \gamma g\right) \cap H=\emptyset$. Hence the equation $g^{-1} y g=y$ implies that

$$
\pi_{H}(y)=g^{-1} \beta g=\beta .
$$

Therefore, $g \beta=\beta g$ and $g \gamma=\gamma g$. Hence the result follows.

Lemma 3.2. Let $F$ be a field and $G$ be a group. Let $g$ be a non-central element of G. If $F G=F C_{G}(g) \oplus M$, then

$$
C_{F G}(g)=F C_{G}(g) \oplus C_{M}(g) .
$$

Proof. Let $\alpha=\alpha_{1}+\alpha_{2}$ be an element of $C_{F G}(g)$, where $\alpha_{1} \in F C_{G}(g)$ and $\alpha_{2} \in M$. Then $g^{-1} \alpha g=\alpha$ implies that $\alpha_{2} \in C_{M}(g)$.

Lemma 3.3. Let $F$ be a finite field of characteristic $p$ and $G$ be a finite $p$-group with the lower central series

$$
G=\gamma_{1}(G) \supseteq \gamma_{2}(G) \supseteq \ldots \supseteq \gamma_{c-1}(G) \supseteq \gamma_{c}(G) \supseteq \gamma_{c+1}(G)=\{e\} .
$$

Let $g$ be a non-central element of $G$. Assume that $\left\{e=c_{1}, c_{2}, \ldots, c_{l}\right\}$ is a transversal of $\gamma_{c}(G)$ in $\gamma_{c-1}(G)$ and $n_{i}=\left[\gamma_{c}(G):\left\langle\left(c_{i}, g\right)\right\rangle\right]$. Then

$$
\left|C_{F \gamma_{c-1}(G)}(g)\right|=|F|^{\left|\gamma_{c}(G)\right|+\sum_{i=2}^{l} n_{i}} .
$$

Proof. Note that $C_{F \gamma_{c-1}(G)}(g)=C_{F \gamma_{c}(G)}(g) \oplus C_{M}(g)$, as an $F$-module, where $M=\bigoplus_{i=2}^{l} c_{i} F \gamma_{c}(G)$. If $y=c_{2} y_{2}+\ldots+c_{l} y_{l} \in C_{F \gamma_{c-1}(G)}(g)$, where $y_{i} \in F \gamma_{c}(G)$, then $g^{-1} y g=y$ implies that

$$
c_{2} y_{2}+c_{3} y_{3}+\ldots+c_{l} y_{l}=c_{2}\left(c_{2}, g\right) y_{2}+\ldots+c_{l}\left(c_{l}, g\right) y_{l} .
$$


Hence $y_{i}=\left(c_{i}, g\right) y_{i} \forall 2 \leq i \leq l$. Let $\left\{e=t_{i 1}, t_{i 2}, \ldots, t_{i n_{i}}\right\}$ be a transversal of the subgroup $\left\langle\left(c_{i}, g\right)\right\rangle$ in $\gamma_{c}(G)$. Write $y_{i}=y_{i 1}+t_{i 2} y_{i 2}+\ldots+t_{i n_{i}} y_{i n_{i}}$, where $y_{i j} \in F\left\langle\left(c_{i}, g\right)\right\rangle \forall 1 \leq j \leq n_{i}$. Now the equation $y_{i}=\left(c_{i}, g\right) y_{i}$ implies that $y_{i j}=\alpha_{j}\left\langle\widehat{\left(c_{i}, g\right)}\right\rangle$, for some $\alpha_{j} \in F$. Thus for each $i, y_{i}$ has $|F|^{n_{i}}$ choices and so $\operatorname{dim}_{F}\left(C_{M}(g)\right)=\sum_{i=2}^{l} n_{i}$. Hence $\left|C_{F \gamma_{c-1}(G)}(g)\right|=|F|^{\left|\gamma_{c}(G)\right|+\sum_{i=2}^{l} n_{i}}$.

Proof of Theorem 1.2: If $G$ is a nilpotent group of class 2, from above lemma we get that $\left|C_{F G}(g)\right|=|F|^{\left|G^{\prime}\right|+\sum_{i=2}^{l} n_{i}}$. Hence

$$
\left|C_{g}\right|=|F|^{|G|-\left(\left|G^{\prime}\right|+\sum_{i=2}^{l} n_{i}\right)} .
$$

In particular, if $G$ is a non-abelian group of order $p^{3}$, then nilpotency class of $G$ is 2 . Theorem 1.3 can be derived from Theorem 1.2 directly. One can also use the following lemma to prove Theorem 1.3.

Lemma 3.4. Let $F$ be a finite field of characteristic $p$. Let $G$ be a finite p-group with the lower central series

$$
G=\gamma_{1}(G) \supseteq \gamma_{2}(G) \supseteq \ldots \supseteq \gamma_{c-1}(G) \supseteq \gamma_{c}(G) \supseteq \gamma_{c+1}(G)=\{e\} .
$$

Let $g$ be a non-central element in $\gamma_{c-1}(G) \backslash \gamma_{c}(G)$. If $\left\{e=d_{1}, d_{2}, \ldots, d_{l}\right\}$ is a transversal of $C_{G}(g)$ in $G$ and $m_{i}=\left[C_{G}(g):\left\langle\left(d_{i}, g\right)\right\rangle\right]$, then

$$
\left|C_{F G}(g)\right|=|F|^{\left|C_{G}(g)\right|+\sum_{i=2}^{l} m_{i}} .
$$

Proof. By Lemma 3.2, $C_{F G}(g)=F C_{G}(g) \oplus C_{M}(g)$ as an $F$-module, where $M=$ $d_{2} F C_{G}(g) \oplus d_{3} F C_{G}(g) \oplus \ldots \oplus d_{l} F C_{G}(g)$. Let $x=\sum_{i=2}^{l} d_{i} x_{i} \in C_{M}(g)$. Then the equation $g^{-1} x g=x$ implies that

$$
\left(d_{i}, g\right) x_{i}=x_{i} \forall 2 \leq i \leq l .
$$

Since $\gamma_{c}(G) \subseteq C_{G}(g),\left\langle\left(d_{i}, g\right)\right\rangle$ is a subgroup of $C_{G}(g)$ and so each $x_{i}$ has $|F|^{m_{i}}$ choices. Therefore, $\left|C_{F G}(g)\right|=|F|^{\left|C_{G}(g)\right|+\sum_{i=2}^{l} m_{i}}$.

Corollary 3.5. Let $F$ be a finite field of characteristic $p$ and $G$ be a finite $p$ group. Assume that $g$ is a non-central element of $G$ such that $G^{\prime} \subseteq C_{G}(g)$. If $\left\{e=d_{1}, d_{2}, \ldots, d_{l}\right\}$ is a transversal of $C_{G}(g)$ in $G$ and $m_{i}=\left[C_{G}(g):\left\langle\left(d_{i}, g\right)\right\rangle\right]$, then

$$
\left|C_{F G}(g)\right|=|F|^{\left|C_{G}(g)\right|+\sum_{i=2}^{l} m_{i}} .
$$


Proof. Since $G^{\prime} \subseteq C_{G}(g)$, the equation $g^{-1} x g=x$ implies (1). Thus the result follows.

Proof of Theorem 1.3: Since $G$ is a group of order $p^{3}$, we have that $G^{\prime}$ is a central subgroup of order $p$ and so for any non-central element $g \in G,\left|C_{G}(g)\right|=p^{2}$. Therefore, $\frac{G}{C_{G}(g)}$ is a cyclic group of order $p$. If $\left\{e, h, h^{2}, \ldots, h^{p-1}\right\}$ is a transversal of $C_{G}(g)$ in $G$, then $\left|\left\langle\left(h^{i}, g\right)\right\rangle\right|=p$. Hence $m_{i}=p \forall 2 \leq i \leq p$. Therefore, $C_{F G}(g)=$ $|F|^{p^{2}+(p-1) p}$ and the result follows.

\section{Class size of elements of groups of order $p^{4}$ and $p^{5}$ in the normalized unit group}

If nilpotency class of a finite $p$-group $G$ is 2 , then the size of the conjugacy class of elements of $G$ in $V(F G)$ can be obtained from Theorem 1.2. Let $p$ be an odd prime. In this section, the length of the conjugacy class of elements of $G$ in $V(F G)$ has been obtained, when $G$ is a group of order $p^{4}$ or $p^{5}$ such that nilpotency class of $G$ is 3 .

Let $G$ be a non-abelian group of order $p^{4}$ with nilpotency class 3. Then $\left|G^{\prime}\right|=p^{2}$ and

$$
\frac{G}{G^{\prime}} \cong C_{p} \times C_{p}
$$

If $\frac{G}{G^{\prime}}=\left\langle g G^{\prime}\right\rangle \times\left\langle h G^{\prime}\right\rangle$, then any commutator $\left(g_{1}, g_{2}\right)$ of $G$ can be written as $(g, h)^{l} z$, where $l \in \mathbb{Z}^{+}$and $z \in \gamma_{3}(G)$. Since $\left|\gamma_{3}(G)\right|=p$, for any $k \in G$, either $G^{\prime} \subseteq C_{G}(k)$ or $G^{\prime} \cap C_{G}(k)=\gamma_{3}(G)$. So, if $G^{\prime} \subseteq C_{G}(g)$, then $h^{p} \in G^{\prime} \subseteq C_{G}(g)$ and by Lemma $2.4,\left(h^{p}, g\right)=e$ implies that $(h, g)^{p}=e$. Further, if $G^{\prime} \cap C_{G}(g)=\gamma_{3}(G)$, then $g^{p} \in \gamma_{3}(G)$ and hence $\left(g^{p}, h\right)=(g, h)^{p}=e$. Since $G^{\prime}$ is abelian, one can conclude that order of each commutator is $p$ and so $G^{\prime} \cong C_{p} \times C_{p}$. Now we give a proof of Theorem 1.4 :

Proof of Theorem 1.4: Let $g$ be a non-central element of $G$. For an element $h \in G, h^{-1} g h=g(g, h)$. Thus the length of the conjugacy class of $g$ in $G$ is at most $p^{2}$ and so $\left|C_{G}(g)\right|=p^{2}$ or $p^{3}$. Since $G^{\prime}$ is elementary abelian $p$-group, Corollary 2.5 implies that $\left|C_{g}\right|=|F|^{(p-1)^{2} p(p+1)}$, when $\left|C_{G}(g)\right|=p^{2}$ and $\left|C_{g}\right|=|F|^{(p-1)^{2} p^{2}}$, when $\left|C_{G}(g)\right|=p^{3}$.

Let $G$ be a non-abelian group of order $p^{5}$ with nilpotency class 3 . Then order of $G^{\prime}$ can be $p^{2}$ or $p^{3}$. Since $\frac{G}{G^{\prime}}$ is not cyclic, it can be isomorphic to either an elementary abelian group or $C_{p^{2}} \times C_{p}$. The following result provides the class 
length of elements of $G$ in $V(F G)$, when $\frac{G}{G^{\prime}}$ is elementary abelian. Note that if $G$ is a $p$-group of class 3 such that $G^{\prime}$ is abelian and $\frac{G}{G^{\prime}}$ is elementary abelian, then $\exp \left(\gamma_{3}(G)\right)$ is $p$; for if $g_{1} \in G$ and $g_{2} \in G^{\prime}$, we have $\left(g_{1}, g_{2}\right) \in \gamma_{3}(G)$ and then $\left(g_{1}, g_{2}\right)^{p}=\left(g_{1}^{p}, g_{2}\right)\left(\left(g_{1}, g_{2}\right), g_{1}\right)^{\left(\begin{array}{l}p \\ 2\end{array}\right)}=e$.

Proof of Theorem 1.5: It is known that a non-abelian group of order $p^{5}$ is metabelian. If $\left|G^{\prime}\right|=p^{2}$, then $\left|C_{G}(g)\right|$ can be $p^{3}$ or $p^{4}$. Further, since $\frac{G}{G^{\prime}}$ is elementary abelian $p$-group, using Lemma 2.4, one can easily check that $G^{\prime}$ is elementary abelian. Therefore, from Corollary 2.5, we have that $\left|C_{g}\right|=|F|^{(p-1)^{2} p^{3}}$, when $\left|C_{G}(g)\right|=p^{4}$ and $\left|C_{g}\right|=|F|^{(p-1)^{2} p^{2}(p+1)}$, when $\left|C_{G}(g)\right|=p^{3}$.

Now suppose that $\left|G^{\prime}\right|=p^{3}$ and $\left|\gamma_{3}(G)\right|=p^{2}$. Then the size of the centralizer of any non-central element can be either $p^{3}$ or $p^{4}$. Since $\gamma_{3}(G) \cong C_{p} \times C_{p}$, one can easily check that $G^{\prime}$ is elementary abelian and hence the result can be obtained from Corollary 2.5.

Further, assume that

$$
\frac{G}{G^{\prime}} \cong C_{p^{2}} \times C_{p} .
$$

Then $G^{\prime}$ is isomorphic to either $C_{p^{2}}$ or $C_{p} \times C_{p}$. We need the following lemma in order to prove Theorem 1.6:

Lemma 4.1. Let $F$ be a finite field of characteristic $p$. Let $G$ be a finite p-group and $H$ be a normal subgroup of $G$ such that $\frac{G}{H}$ is a cyclic group of order $p^{n}$. Then for any $g \in G \backslash H$,

$$
\left|C_{F G}(g)\right|=\left|C_{F H}(g)\right|^{p^{n}} .
$$

Proof. Since $\frac{G}{H}=\langle g H\rangle$ is a cyclic group of order $p^{n}$, we have that $F G=$ $\bigoplus_{i=0}^{p^{n}-1} g^{i} F H$. Let $\alpha=\sum_{i=0}^{p^{n}-1} g^{i} \alpha_{i}$, where $\alpha_{i} \in F H$, be an element of $C_{F G}(g)$. Since $H$ is a normal subgroup of $G, g^{-1} \alpha g=\alpha$ implies that $\alpha_{i} \in C_{F H}(g) \forall 0 \leq i \leq p^{n}-1$. Therefore, $\left|C_{F G}(g)\right|=\left|C_{F H}(g)\right|^{p^{n}}$.

Proof of Theorem 1.6: Since $\left|G^{\prime}\right|=p^{2}$, the size of the centralizer of any noncentral element $g$ in $G$ is $p^{3}$ or $p^{4}$. In particular, if $g \in G^{\prime}$, then the length of the conjugacy class of $g$ in $G$ is $p$ and so $\left|C_{G}(g)\right|=p^{4}$. Note that if $G^{\prime} \cong C_{p} \times C_{p}$, the result can be obtained from Corollary 2.5. So, consider the case when $G^{\prime} \cong C_{p^{2}}$. Suppose for some $g \in G,\left|C_{G}(g)\right|=p^{4}$. Hence $\left|\frac{G}{C_{G}(g)}\right|=p$ and so for any $h \in$ $G, h^{p} \in C_{G}(g)$. Therefore, $\left(h^{p}, g\right)=e$ and hence from Lemma $2.4,(h, g)^{p}=e$. So, 
$\left(g^{p}, h\right)=(g, h)^{p}=e$. Thus $g^{p} \in Z(G)$ and from Corollary 2.3, one can obtain that $\left|C_{g}\right|=|F|^{p^{3}(p-1)^{2}}$.

Next suppose that $\left|C_{G}(g)\right|=p^{3}$ and so $g \in G \backslash G^{\prime}$. Write $\frac{G}{G^{\prime}}=\left\langle g G^{\prime}\right\rangle \times\left\langle h G^{\prime}\right\rangle$, for some $h \in G$. Note that for any non-central element $g \in G$, either $G^{\prime} \subseteq C_{G}(g)$ or $G^{\prime} \cap C_{G}(g)=\gamma_{3}(G)$. Assume that $o(g) \bmod G^{\prime}$ is $p$ and $G^{\prime} \cap C_{G}(g)=\gamma_{3}(G)$. Now $g^{p} \in \gamma_{3}(G)$ and so $\left(g^{p}, h\right)=e$ implies that $(g, h)^{p}=e$. Therefore, $\exp \left(G^{\prime}\right)=p$, which is a contradiction. Thus $G^{\prime} \subseteq C_{G}(g)$. Further, if $h^{p} \in C_{G}(g)$, then $\exp \left(G^{\prime}\right)=$ $p$. Therefore, $h^{p} \notin C_{G}(g)$. As $h^{p^{2}} \in C_{G}(g)$, we have $F G$ as $F C_{G}(g)$-module with the elements $\left\{h^{i} \mid 0 \leq i \leq p^{2}-1\right\}$ as a free basis. If $(i, p)=1$, then from Lemma 2.4 , we get that $o\left(h^{i}, g\right)=p^{2}$. Otherwise, $o\left(h^{i}, g\right)$ is $p$. Therefore, from Corollary $3.5,\left|C_{F G}(g)\right|=|F|^{p^{2}(3 p-2)}$.

Further, assume that $o(g) \bmod G^{\prime}$ is $p^{2}$. Let $H=\left\langle G^{\prime}, h\right\rangle$. From Lemma 4.1, we have that $\left|C_{F G}(g)\right|=\left|C_{F H}(g)\right|^{p^{2}}$. Now by Lemma 3.1, $C_{F H}(g)=C_{F G^{\prime}}(g) \oplus C_{N}(g)$, where $N=\bigoplus_{i=1}^{p-1} h^{i} F G^{\prime}$. If $\alpha=\sum_{i=1}^{p-1} h^{i} \alpha_{i}$ is an element of $C_{N}(g)$, then $\left(h^{i}, g\right) \alpha_{i}=$ $\alpha_{i} \forall 1 \leq i \leq p-1$. Now Lemma 2.4 implies that for each $i,\left(h^{i}, g\right)$ is the generator of $G^{\prime}$ and hence we get that $\alpha_{i}=\beta_{i} \widehat{G^{\prime}}$, where $\beta_{i} \in F$. Hence $\left|C_{N}(g)\right|=|F|^{p-1}$. Observe that in this case $G^{\prime} \nsubseteq C_{G}(g)$, since then $C_{G}(g)=\left\langle G^{\prime}, g\right\rangle$ and so $\left|C_{G}(g)\right|=$ $p^{4}$. Now $\left\{\left(h^{i}, g\right) \mid 0 \leq i \leq p-1\right\}$ is a transversal of $\gamma_{3}(G)$ in $G^{\prime}$. Since $\left|\left\langle\left(\left(h^{i}, g\right), g\right)\right\rangle\right|=$ $p$, for each $1 \leq i \leq p-1$, it follows from Lemma 3.3 that $\left|C_{F G^{\prime}}(g)\right|=|F|^{p+(p-1)}$. Thus $\left|C_{F G}(g)\right|=|F|^{p^{2}(3 p-2)}$ and so $\left|C_{g}\right|=|F|^{p^{2}\left(p^{3}-3 p+2\right)}$.

\section{References}

[1] A. A. Bovdi and I. I. Hripta, Normal subgroups of the multiplicative group of a ring, Mat. Sb. (N.S.), 87(129) (1972), 338-350.

[2] A. A. Bovdi and S. V. Mihovski, Conjugacy classes in group algebras of $p$ groups, C. R. Acad. Bulgare Sci., 56(1) (2003), 5-8.

[3] A. A. Bovdi and C. Polcino Milies, Conjugacy classes of the group of units in group algebras of finite p-groups, Euroconference on Algebra and Representation Theory (Constantza, 2000), An. Ştiinţ. Univ. Ovidius Constanţa Ser. Mat., 8(2) (2000), 1-12.

[4] V. Bovdi and A. L. Rosa, On the order of the unitary subgroup of a modular group algebra, Comm. Algebra, 28(4) (2000), 1897-1905.

[5] A. Bovdi, L. G. Kovács and S. Mihovski, On the orders of conjugacy classes in group algebras of p-groups, J. Aust. Math. Soc., 77(2) (2004), 185-189. 
[6] D. B. Coleman, On the modular group ring of a p-group, Proc. Amer. Math. Soc., 15 (1964), 511-514.

[7] M. A. Rao and R. Sandling, Vanishing orbit sums in group algebras of p-groups, Groups '93 Galway/St. Andrews, Vol. 2, London Math. Soc. Lecture Note Ser., Cambridge Univ. Press, Cambridge, 212 (1995), 507-511.

Surinder Kaur and Manju Khan (Corresponding Author)

Department of Mathematics

Indian Institute of Technology Ropar

Nangal Road

Rupnagar - 140 001, India

e-mails: surinder.kaur@iitrpr.ac.in (S. Kaur)

manju@iitrpr.ac.in (M. Khan) 